\title{
LA INVESTIGACIÓN EN EL PROCESO DE FORMACIÓN UNIVERSITARIA DEL COMUNICADOR SOCIAL
}

\section{RESEARCH IN THE UNIVERSITY TRAINING PROCESS OF THE SOCIAL COMMUNICATOR}

Cynthia Katherine Icaza Cárdenas, Lcda. Licenciada en Ciencias de la Comunicación Social (Ecuador).

Productora e Investigadora Científica. Estudiante del Máster en Comunicación Social itinerario Investigación en la Universidad de Almería, España. katita_ckic@ @hotmail.com

Christian Josué Riquero Pincay, Lcdo.

Licenciado en Ciencias de la Comunicación Social (Ecuador). Productor e Investigador Científico. Estudiante del Máster en Comunicación Social itinerario Investigación en la Universidad de Almería, España. crisjosuk @hotmail.com

Delia Silvia Peña Hojas, Mgs. Magíster en Gerencia Educativa (Ecuador). Estudiante de Doctorado de la Universidad de La Habana, Cuba. Docente investigadora científica de la Facultad de Ciencias Médicas en la Universidad Católica de Santiago de Guayaquil, Ecuador. dphojas@hotmail.com

Isabel Leonor Marín Esteves, Mgs.

Magíster en Docencia Universitaria e Investigación Educativa (Ecuador). Docente de la Facultad de Comunicación Social en la Universidad de Guayaquil, Ecuador. imarinesteves@gmail.com

\section{ARTÍCULO DE INVESTIGACIÓN}

Recibido: 27 de agosto de 2018.

Aceptado: 30 de noviembre de 2018 .

ISSN: 1390-9320, Edición Especial, diciembre 2018 


\section{RESUMEN}

El presente estudio sobre la investigación en el proceso de formación universitaria del Comunicador Social de la Universidad de Guayaquil, tiene como objetivo determinar el grado de producción en investigación que tienen los estudiantes y el desarrollo de competencias investigativas necesarias para generar conocimientos que les posibiliten enfrentar retos en su ejercicio profesional. Es importante fomentar una cultura investigativa mediante procesos de sensibilización, reflexión, experimentación, análisis y crítica, donde tanto el currículo como el rol del docente juegan un papel relevante para impulsar la actividad productiva asociada a la investigación científica. El método aplicado es el analítico-sintético; y como técnica la encuesta. Los resultados mostraron que a pesar de que se trate de promover la investigación acorde a las normativas jurídicas de la educación superior tanto a nivel nacional como institucional, todavía no se erradican las insuficiencias y son visibles, al no existir publicaciones científicas. Se plantea una propuesta de cambio en la formación académica que involucre la investigación formativa de los Comunicadores de la Facultad de Comunicación Social de la Universidad de Guayaquil (FACSO).

Palabras Clave: investigación formativa, comunicador, cultura, publicaciones, indagación

\section{ABSTRACT}

The present study on research in the process of university education of the Social Communicator of the University of Guayaquil, aims to determine the degree of research production that students have and the development of research skills necessary to generate knowledge that will enable them to face challenges in his professional practice. It is important to promote a research culture through processes of awareness, reflection, experimentation, analysis and criticism, where both the curriculum and the role of the teacher play an important role in promoting the productive activity associated with scientific research. The applied method is the analytical-synthetic; and as a technique the survey. The results showed that in spite of the fact that it is a question of promoting research according to the legal norms of higher education both nationally and institutionally, insufficiencies are still not eradicated and are visible, since there are no

ISSN: 1390-9320, Edición Especial, diciembre 2018 
scientific publications. A proposal of change in the academic formation that involves the formative research of the Communicators of the Faculty of Social Communication of the University of Guayaquil (FACSO) is proposed.

Keywords: formative research, communicator, culture, publications, inquiry.

\section{INTRODUCCIÓN}

El Ecuador en el ámbito de la comunicación ha sufrido cambios tanto sociales, económicos como educativos desde que se empezó a impulsar a que se realicen investigaciones científicas en los diferentes cuerpos legales, muchos profesionales de la comunicación comenzaron a desarrollar publicaciones investigativas.

En cuanto a la formación de los Comunicadores Sociales el autor expresa "Investigar en el pregrado es prepararse para la vida" (Echeverri,2003, 95)".

Esta es la principal razón por la cual se plantea que debe existir la suficiente preparación investigativa dentro de la malla curricular para que se pueda estimular el pensamiento crítico a través de fases del aprendizaje, donde se vitalice y se luche contra la memorización y se contribuya con la formación de profesionales competentes en el área de la Comunicación Social.

\section{REVISIÓN TEÓRICA}

\subsection{La investigación formativa para el aprendizaje académico}

La investigación formativa "es un proceso de aprender haciendo, en el cual se involucra al estudiante para que de manera activa construya su propio conocimiento, a través de la búsqueda de información, reflexión y del análisis" (Ríos,2014, 175).

Según este autor la investigación formativa se da por medio del procedimiento de aprender realizando investigaciones que son divulgadas a la sociedad, se lo puede 
considerar como una búsqueda de conocimientos que están caracterizados por la creación de actos que sirvan para la innovación de ideas y de métodos.

El propósito de la investigación formativa es hacer que los futuros comunicadores sociales de la Universidad de Guayaquil cuenten con un ambiente adecuado y formen una cultura de investigación.

La investigación se basa en tres estrategias que son: el levantamiento de información, la estrategia que está orientada a la recolección de datos reales para reconocer los problemas y dar diferentes posibles soluciones a través de las observaciones, cuestionarios y entrevistas.

La importancia de estudio radica en contribuir con el desarrollo de formación de los comunicadores sociales de la Universidad de Guayaquil y que se reconozca la importancia que tiene la aplicación de la investigación en el proceso de formación desde las aulas de clases.

\subsection{Las cualidades que adquiere el estudiante dentro del proceso de la investigación formativa}

- La investigación formativa busca que los estudiantes tengan las cualidades necesarias para su desarrollo profesional entre estas son:

- Generar diversos conocimientos con la rigurosidad científica en el campo de la ciencia

- Contribuyan al desarrollo de los conflictos de la población.

- Realicen diagnósticos exhaustivos de los fenómenos.

- Complementen sus conocimientos desde otras perspectivas

- Mejoren los procesos de publicaciones científicas

\subsection{Razones de la Investigación Formativa}

La investigación formativa mejora la calidad de la educación por cuatro razones:

ISSN: 1390-9320, Edición Especial, diciembre 2018 
- Desarrollar habilidades cognoscitivas como analíticas además del pensamiento y dar soluciones de los problemas del entorno.

- Familiarizar a los estudiantes con la etapa de investigación científica y los problemas que se plantean en la sociedad.

- Construir tanto en los estudiantes y los docentes una cultura de evaluación de manera permanente de la práctica a través de los procesos investigativos.

- Enseñar a investigar a estudiantes y docentes según diferentes métodos y procesos.

\subsection{Objetivo de la Investigación Formativa}

Los principales objetivos de la investigación formativa son: preparar a los estudiantes a través del desarrollo de actividades que permita comprender y adelantar los procesos de la indagación, formar y estructurar o redefinir publicaciones y formar y transformar a través de prácticas de contenidos científicos para que cumpla el campo de investigación y acción.

La finalidad de la investigación formativa está centrada en desarrollar competencias investigativas a través del pensamiento crítico y metacognitivo.

\subsection{Propósito de la Investigación Formativa}

El principal propósito está ajustado al desarrollo de competencias a través del pensamiento tanto analítico, reflexivo, crítico y metacognitivo que permite al profesor y al estudiante enseñar a explorar y obtener solución a los problemas que ocurren a los alrededores y de esta manera una cultura investigativa.

\subsection{Características de la Investigación Formativa}

Las principales características de la investigación son:

- Su intención curricular que contribuyen con el desarrollo de los procesos de enseñanza-aprendizaje vinculado con el conocimiento.

- Estos tienen objetivos curriculares y propósitos curriculares dentro de la formación académica donde se desarrolla la formación científica.

- Esta investigación es dirigida por un docente. 
- Los agentes investigadores son los estudiantes.

\section{Competencias Investigativas en el Comunicador Social}

La competencia investigativa es el motor orientador del conocimiento científico y está integrada por capacidades tales como: manejar críticamente la bibliografía, escoger y delimitar el problema a investigar; diseñar el proceso de la validación, verificación o legitimación de las hipótesis y esta "empieza por la aplicación de los conocimientos, enfatizando en las diferentes esferas involucradas en la actividad investigativa entre las que se destacan las dimensiones epistemológica, metodológica, técnica y social" (Maldonado, 2007,46).

El desarrollo de las competencias investigativas dentro de la Educación Superior permite a las personas brindar soluciones a los problemas profesionales desde la perspectiva científica investigativa. Su formación y desarrollo está condicionado al currículo que maneja las carreras universitarias donde se muestra el interés, las necesidades y la demanda que tiene la sociedad afianzando habilidades para observar, preguntar, argumentar y sistematizar y así gestionar conocimiento.

Para lograr estas competencias se debe poner en práctica estrategias de enseñanza, para que el estudiante se empodere de las herramientas de la investigación y así ponga en práctica los conocimientos en el campo laboral profesional.

\section{Ejes de la Investigación Formativa}

Los ejes de la investigación formativa son:

\subsection{Docencia}

El docente es un agente del proceso educativo formador del estudiante, es por eso la importancia de una correcta capacitación y la aplicación y el manejo de diferentes metodologías didácticas que son empleadas en el aula. 


\subsection{Currículo}

Es el instrumento elemental donde se organizan las experiencias educativas que debe tener el futuro profesional de Comunicación Social, donde se incluye los planes de estudios que están estructurados en ciclos que contiene un sistema de créditos, está sujeto a un modelo pedagógico centrado en el estudiante edifique sus conocimientos y se logre estimular su imaginación, creatividad, capacidad para reflexionar y analizar el objeto.

Este currículo debe ser integral enlazado con materias de importancia para la formación académica de manera flexible de manera sólida y rigurosa.

En la malla curricular se tiene contenidos de signaturas de nivel básico como aplicado estas son sobre teoría y método científico, investigación, desarrollo de tesis entre otras y de esta manera la universidad concibe condiciones que permite la formación del estudiante investigador que es capaz de lograr un acercamiento a la realidad que se va a buscar.

\section{Estrategia para promover la investigación formativa en la Educación Superior}

La investigación formativa en le Educación Superior facilita el desarrollo de diferentes competencias, habilidades de aprendizaje necesarias para el ejercicio profesional de la comunicación , "da como resultado el estímulo del aprendizaje autónomo, desarrolla una estructura mental de manera ordenada, desarrolla el pensamiento crítico, propositivo y lógico, el desarrollo de la indagación, clasificación, análisis de interpretación de la información recolectada, estimula el rigor académico (Guerrero, 2007, p. 5) y (Gómez, 2013, p. 3)

\subsection{La investigación en el ámbito pedagógico}

"La formación para la investigación es el conjunto de acciones orientadas a la apropiación y desarrollo de conocimientos, habilidades y actitudes para que estudiantes y profesores puedan desempeñar de manera eficientes actividades de investigación, tecnología, innovación, esto forma un modelo formativo” (Guerrero,2007, p. 190). 
Esto da a entender que la investigación favorece el progreso de los futuros comunicadores sociales porque permite obtener las instrucciones necesarias para realizar investigaciones científicas, pero esta no puede realizarse si no se cuenta con infraestructura y recursos tanto físicos como virtuales que hagan posible una actividad de investigación académicamente competitiva, para esto también se requiere de docentes dedicados que estén altamente calificados además que cuenten con la experiencia que les permita desarrollar significativas investigaciones con los estudiantes.

Los estudiantes universitarios deben aprender realizando investigaciones científicas junto al docente investigador para que de esta manera se logre transmitir a los estudiantes la pasión por realizar publicaciones científicas que contribuya a la ciencia y tecnología.

\section{Los roles de los alumnos en la Investigación Formativa}

Para lograr construir una sociedad de conocimiento, la calidad de la Educación Superior debe está coordinada y asociada a la investigación esta se relaciona de dos maneras tanto enseñar a investigar y realizar investigaciones y "esta estrategia pedagógica se realiza a través de la investigación formativa, transforma e implica diversas maneras de aprendizaje por medio del descubrimiento y construcción" (Restrepo, 2003, p.8).

El autor manifiesta que los estudiantes deben aprender a hacer investigaciones científicas por medio de las exploraciones, las cuales permitirán dar posibles soluciones al acontecer mundial.

\section{Los pilares importantes para cumplir con los estándares internacionales de investigación}

La universidad, la empresa y el Estado son los encargados de la consolidación del proceso de la investigación, es necesario que definan sus metas de producciones investigativas, se la puede considerar "un organismo de servicio público e interés nacional, autónoma y descentralizada en el que se prepara a los futuros profesionales que llevan a cabo investigaciones y labores de extensión" (Souza,2005, p.18). 
Como lo dice el autor la universidad debe de ser creada como un impulsador del desarrollo económico y social de un país, cuya misión principal es la investigación para que así se contribuya con la formación de los futuros profesionales, también se lo considera como un espacio de transformación donde la malla curricular se ajusta a un perfil de egreso de un profesional investigador.

El compromiso de la Universidades con la sociedad es permitir construir la creación de conocimientos la cuales deben ser compartidas en las actividades con la comunidad, donde se les brinde las herramientas elementales para el crecimiento profesional.

El objetivo que se quiere lograr es motivar a los estudiantes de Comunicación Social a producir investigaciones científicas y que los docentes utilicen a la investigación como herramienta pedagógica que sirva para tener un desempeño exitoso en el campo laboral. La esencia de las universidades es la investigación, que tienen como misión formar profesionales dedicados a la producción de ciencia y tecnología.

La institución de Educación Superior debe cumplir cabalmente con la misión de transformar a la realidad social, para esto se requiere profesores que sean éticos, capaces y que estén preparados, no solamente que conozcan y manejen contenido científico investigativo de su campo disciplinar, sino que además cuente con la formación profesional para examinar los conflictos y las necesidades de la sociedad y de la ciencia, que permita guiar a los estudiantes de manera efectiva para que sepa determinar los puntos de interés en lo cual se debe investigar.

Desde el eje de la investigación formativa dentro de la Universidad se promueve la interdisciplinariedad y la transdisciplinariedad incorporada en el desarrollo de actitudes, capacidades y acciones intelectuales que fortalece la aplicación de fundamentos científicos y el desarrollo del pensamiento creativo en la población.

\section{Cultura Investigativa}

La cultura investigativa se desarrolla mediante el proceso educativo, la extensión, la vinculación con el entorno por medio de la investigación, estas se deben ejecutar con el apoyo también de los actores tanto intra como extra institucionales que los conforman

ISSN: 1390-9320, Edición Especial, diciembre 2018 
los directivos, docentes, estudiantes y miembros de la comunidad o con los actores comunitarios y se considera "insuficiente cuando no se posibilita la producción científica, se debe promover desde las aulas" (Leal ,2009, p.2).

Otro autor considera que "en la universidad la cultura no se inicia con sistemas, sino con profesores individuales que se integren en equipos, grupos, comités, centro de investigación y desarrollo tecnológico, así como redes que construyan el sistema de investigación" (Bracho, 2012, p.1).

Estos autores ayudan a entender que la cultura investigativa empieza desde las aulas cuando se logra fortalecer, promover, formar investigadores que desarrollan diferentes líneas de investigación donde analizan lo que sucede alrededor del estudiante, lo cual le permite ubicarse en un nivel alto de investigación y por lo tanto realizar temas interesantes de publicaciones científicas.

\subsection{Desarrollo de la Cultura de Investigación en la Educación Superior}

La investigación está compuesta por tres dominios:

- La misión de la Universidad es: enseñar, investigar y la vinculación con la comunidad.

- Los docentes de la Universidad deben tener conocimientos, habilidades, valores y actitud para dirigir a la investigación y estos deben tener disponibilidad de tiempo para la práctica investigativa.

- La institución debe tener disposición para habilitar o facilitar la búsqueda de información.

Es necesario que los centros de Educación Superior cuenten con docentes dedicados a la investigación, porque "El docente investigador tiene el poder que le confiere el ser constructor y creador de conocimientos a partir de sus propias investigaciones, estos deben promover la apertura de espacios académicos, donde la investigación sea la base de la docencia” (Hernández, 2009, p. 6). 
Según el autor el docente universitario tiene la capacidad de dirigir y orientar en los procesos de investigación y ser creadores de conocimientos a través de la experiencia directa y sistematizada.

Los docentes que implementas en las prácticas pedagógicas de los futuros Comunicadores Sociales la investigación en las aulas de clases logran mejorar las habilidades en los procesos científicos contribuyen a la resolución de los problemas y el entendimiento de la ciencia.

\section{Importancia del Docente Investigador}

La importancia del docente investigador establece el desarrollo de competencias con el fin de lograr la adquisición de una personalidad que maneje los fundamentos de la ciencia.

- El docente investigador tiene las siguientes características:

- El docente investigador es observador.

- El docente investigador sabe cómo formular preguntas este debe tener conocimiento del manejo del proceso tanto de enseñanza y de aprendizaje.

- El docente investigador es un aprendiz porque a través de la observación aprende de sus alumnos.

\subsection{Competencias Formativa del Docente Investigativo}

El docente es un investigador por excelencia se maneja bajo competencias investigativas que:

- Permite comprender el significado, la importancia y las complicaciones que se pueden presentar en la investigación formativa

- Nos permite observar, registrar, interpretar, analizar, describir contextos y textos acerca del problema y esto contribuirá a proponer soluciones a los problemas detectados utilizando diferentes teorías y métodos de investigación.

ISSN: 1390-9320, Edición Especial, diciembre 2018 


\section{Currículo clave del desarrollo del campo investigativo}

La producción investigativa debe estar incluida en la formación de los estudiantes y para ello el currículo de la carrera debe estar diseñados a partir de asignaturas que aporten a la investigación de manera pragmática, el desarrollo de esta investigación "requiere que existan condiciones objetivas en las instituciones educativas, un currículo flexible, abierto y dinámico que acepte una diversidad de competencias, valores que permitan la articulación entre los trabajos investigativos y docente y así lograr el progreso de los alumnos" (Cerda, 2007, p. 39).

Es por esta razón que se plantea que debe existir una suficiente preparación dentro de la malla curricular en los estudiantes de la carrera de Comunicación Social de la Universidad de Guayaquil, donde se les dé tanto la parte teórica como práctica que le permitirá realizar publicaciones científicas y contribuya con el fortalecimiento del ámbito académico. El currículo es el conjunto sistematizado tanto de teorías, conceptos, objetivos y contenido de las asignaturas, metodología y criterios de evaluación que definen la carrera universitaria, se considera a este como "un sistema de competencias donde interviene capacidades, actitudes en el desarrollo académico y profesional que están formulados en los diseños curriculares y desarrollados flexiblemente por el alumno" (Arias, 2007, p. 9).

El currículo flexible se basa en el principio de que la educación superior debe centrarse en el aprendizaje de formas y métodos de pensamiento e investigación, bajo un enfoque que rescate y ponga en práctica la formación integral y autónoma en el desarrollo de investigación del estudiante, contando con la participación directa y activa en el diseño de su plan de estudios y en los procesos o educativos, promoviendo el desarrollo, la formación integral y el ejercicio investigativo.

\section{Producción científica Investigativa}

La producción científica que se realiza dentro de las instituciones de educación universitaria se ha vuelto un elemento crucial para el desarrollo de conocimiento y el de las mismas instituciones. Para esta producción se requiere una verdadera difusión que permita a los docentes y estudiantes adquirir conocimientos de normas, reglas que

ISSN: 1390-9320, Edición Especial, diciembre 2018 
permitan realizar los trabajos investigativos como artículos en revistas indexadas y oficiales de las universidades y" se la considera como parte materializada del conocimiento generado" (Piedra, 2007, 33).

La producción científica se deriva de la investigación y su principal objetivo es a divulgación de información científica obtenida en los procesos investigativos.

\section{ANÁLISIS DE RESULTADOS}

La investigación realizada tiene una perspectiva cualitativa, los métodos que se utilizaron fueron teóricos que abarca el análisis y la síntesis y el método bibliográfico. La técnica que se aplicaron para obtener los resultados son las encuestas que contienen diferentes interrogantes que permiten dar soluciones viables para la formación de los Comunicadores de la Facultad de Comunicación Social.

La encuesta se realizó a los estudiantes del séptimo semestre de la carrera de Comunicación Social de la Universidad de Guayaquil en el año 2017 - 2018, dentro de la malla curricular vieron 6 materias relacionadas con la investigación que son: técnicas y métodos de la investigación, periodismo investigativo, investigación de la comunicación, comunicación científica y estadística de la comunicación y elaboración de anteproyecto de titulación que son previas a la realización del trabajo de titulación. La población que se consideró fue de 455 alumnos de la carrera de Comunicación Social de la Universidad de Guayaquil, la cual se tomó una muestra probabilística y se realizó la encuesta a 315.

Los datos que fueron otorgados por la Coordinadora de Investigación de la Facultad de Comunicación Social sobre la cantidad de publicaciones de investigación hasta mayo del 2018 mostraron que los profesores han realizado 25 artículos regionales, 39 ponencias regionales, 5 libros y 1 artículo de alto impacto.

En cuanto a los estudiantes solo se ha realizado un artículo científico a través del fondo competitivo de investigación (FCl). 


\section{DISCUSIÓN}

12. 1 De la encuesta realizada a los 315 estudiantes:

La primera pregunta evaluada a los estudiantes es:

1) ¿Presentan en evento científico un trabajo de investigación?

El cual $97 \%$ de los encuestados expresan que nunca presentan investigaciones científicas y el $3 \%$ que casi nunca, esto muestra que la mayor parte de los estudiantes no tiene interés de desarrollar publicaciones científicas y esto se debe a que los docentes investigadores no despertaron el interés de investigar en los estudiantes de la carrera de Comunicación Social de la Universidad de Guayaquil.

Tabla 1. Presentación de investigación en eventos científicos

\begin{tabular}{cccc}
\hline Valoración & Alternativas & Frecuencia & Porcentaje \% \\
\hline $\mathbf{5}$ & Siempre & 0 & $0 \%$ \\
$\mathbf{4}$ & Casi siempre & 0 & $0 \%$ \\
$\mathbf{3}$ & Algunas veces & 0 & $0 \%$ \\
$\mathbf{2}$ & Casi nunca & 8 & $3 \%$ \\
$\mathbf{1}$ & Nunca & 307 & $97 \%$ \\
\hline & TOTAL & $\mathbf{3 1 5}$ & $\mathbf{1 0 0 \%}$ \\
\hline
\end{tabular}

Fuente: Elaboración propia

ISSN: 1390-9320, Edición Especial, diciembre 2018 
Gráfico 1: Presentan investigaciones en eventos

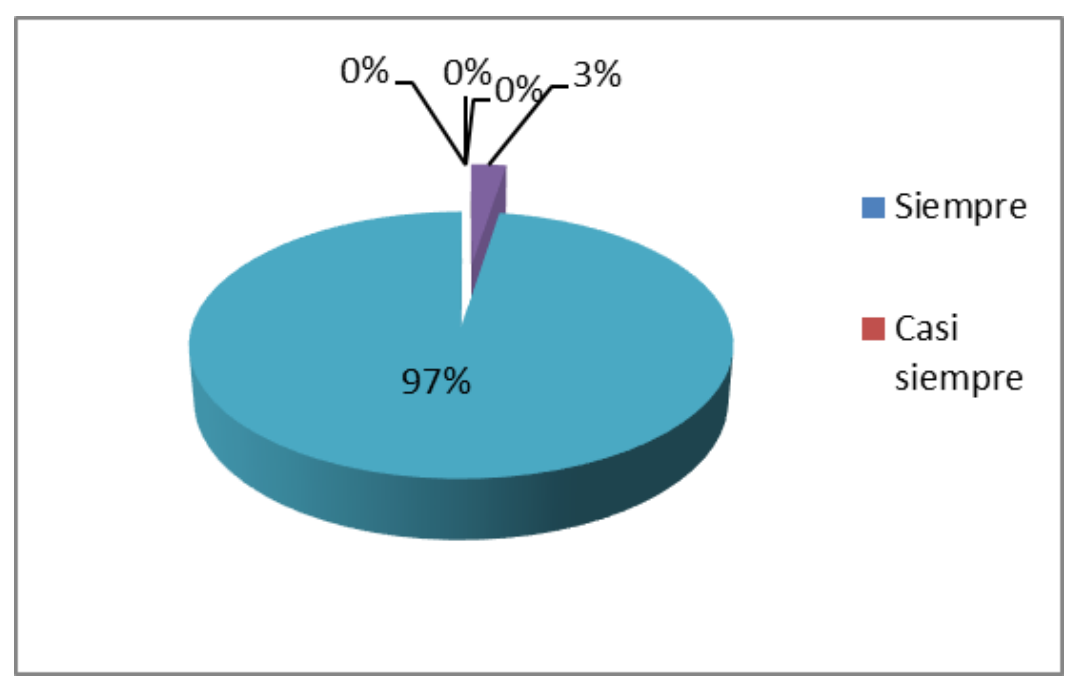

2) ¿EI personal docente lo motiva para realizar investigaciones científicas?

La cual respondió el $66 \%$ de los estudiantes encuestados expresaron que el docente casi nunca los motivas a crear investigaciones científicas, el 19\% que algunas veces y el $15 \%$ casi siempre, estos resultados reflejan que el estudiante no se siente atraído por la investigación y que el docente no cumple con el rol de contribuir con el desarrollo de la formación y esto se ve reflejado porque no existe estadística de investigaciones realizada por los estudiantes de Comunicación Social en la base de datos de la universidad.

Tabla 1. Docentes motivan para realizar investigaciones científicas

\begin{tabular}{cccc}
\hline Valoración & Alternativas & Frecuencia & Porcentaje \% \\
\hline $\mathbf{5}$ & Siempre & 0 & $0 \%$ \\
$\mathbf{4}$ & Casi siempre & 47 & $15 \%$ \\
$\mathbf{3}$ & Algunas veces & 59 & $19 \%$ \\
$\mathbf{2}$ & Casi nunca & 209 & $66 \%$ \\
$\mathbf{1}$ & Nunca & 0 & $0 \%$ \\
- & & &
\end{tabular}

ISSN: 1390-9320, Edición Especial, diciembre 2018 


\begin{tabular}{lll}
\hline TOTAL & 315 & $100 \%$ \\
\hline
\end{tabular}

Fuente: Elaboración Propia

Gráfico 2: Motivación para realizar investigaciones

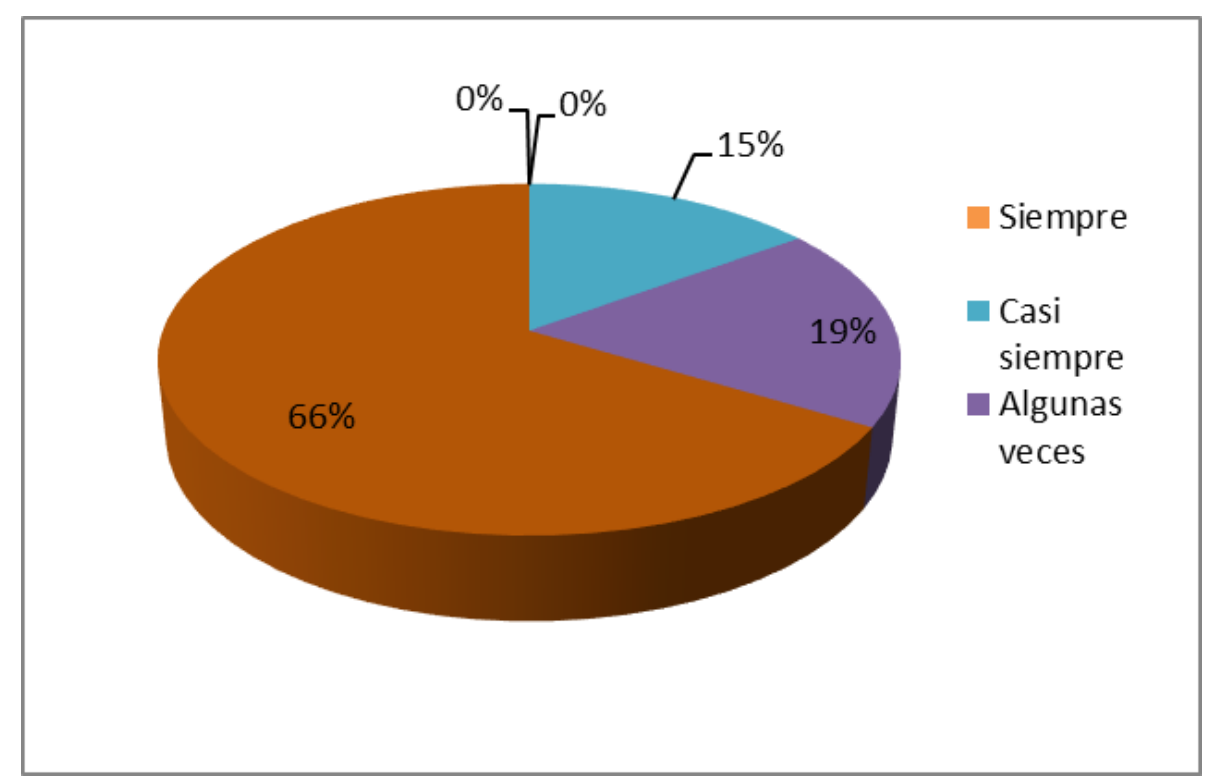

Fuente: Elaboración Propia

El sesgo que puede contar en las encuestas realizadas a los estudiantes del séptimo semestre de la carrera de Comunicación Social este puede tener un mínimo margen de error.

\section{CONCLUSIONES}

La vigente malla curricular de la carrera de Comunicación Social de la Universidad de Guayaquil consta de 7 materias que imparten asignaturas relacionadas con la investigación, se conoce que desde la formación el Comunicador Social debe tener una adecuada preparación y aprender por medio del docente de manera teórica como práctica, esto permitirá eliminar el principal problema existente que es el escaso compromiso de los docentes y estudiantes en realizar investigaciones científicas de alto impacto.

ISSN: 1390-9320, Edición Especial, diciembre 2018 
En la carrera de Comunicación Social de la Universidad de Guayaquil no existe la predisposición y actitud para la realización de investigación por parte de los Comunicadores Sociales y esto se transforma es una mala señal para el presente y el futuro del Ecuador.

Se busca promover a nivel de los estudiantes la generación de publicaciones científicas a través de los docentes, además se realizará los procedimientos necesarios para agilizar los trámites correspondientes que logren que los estudiantes obtengan una ayuda y estímulos económicos necesarios para la producción de investigaciones. El papel de los docentes en el fortalecimiento de los procesos de la formación investigativa se considera una necesidad es por eso que se debe aplicar la investigación como herramienta pedagógica dentro de las aulas de clases de manera teórica como práctica creando contenidos científicos y así formar una cultura investigativa.

El incremento de publicaciones científicas eleva el indicador, que permitirá la acreditación de la carrera por medio de Consejo de evaluación acreditación y aseguramiento de la calidad de la Educación Superior (CEEACES), organismo del Estado encargado de este proceso.

\section{REFERENCIAS BIBLIOGRÁFICAS}

Arias. (2007). Orientaciones Teórico Metodológicas para el Desarrollo del Currículo por Competencias y Flexible. Perú: UNA-PUNO.

Bracho. (2012). Cultura investigativa y producción científica en universidades privadas del municipio Maracaibo del estado Zulia. Recuperado el 13 de 08 de 2018, de http://www.publicaciones.urbe.edu/index.php/REDHECS/article/viewArticle/952/2998

Cerda. (2007). La investigación formativa en el aula: La pedagogía. Bogotá: Magisterio 2007.

Cerda. (2007). La investigación formativa en el aula: La pedagogía como investigación. Bogotá: Magisterio. 
Gómez, B. R. (2013). Fundamentos teóricos de la evaluación por competencias: trazabilidad histórica del concepto. Uni-pluri/versidad. Recuperado el 28 de Agosto de 2018, de https://aprendeenlinea.udea.edu.co/revistas/index.php/unip/article/viewFile/16970/1469 4

González. (2005). El desarrollo de competencias científicas en la educación superior. Medellín: Revista Lectiva.

González, E. (2006). La investigación formativa como una posibilidad para articular las funciones universitarias de la investigación, la extensión y la docencia. . Revista Educación y Pedagogía.

Guerrero M. (2007). Formacion para la investigacion . Studioitas.

Hernández I. (2009). El docente investigador en la formación de profesionales. Revista Católica del norte, 1-21.

Leal,J. (2009). La autonomia del sujeto investigador y la metodologia de investigación. Valencia: Azul Intenso 2da. Edición.

Maldonado, L. F.; Landazábal, D. P.; Hernández, J. C.; Ruiz, Y.; Claro, A.; Vanegas, H. \& Cruz, S. (2007). Visibilidad y formación en investigación. Estrategias para el desarrollo de competencias investigativas. Studiositas, 43- 56. Recuperado el 02 de septiembre de 2018, de Recuperado de http://regweb.ucatolica.edu.co/...O\%20LANDAZABAL1.pdf

Piedra, Y. (2007). Producción científica. Ciencias de la Información La Habana. Instituto de Información científica y tecnológica, Cuba, Vol. 38, No.3 ,33 - 38.

Restrepo G.B. (2003). Conceptos y aplicaciones de la invetsigación formativa y criterios para evaluar la investigación científica en sentido estricto. Bogota: CNA.

ISSN: 1390-9320, Edición Especial, diciembre 2018 
Rios, L. T. (2014). La formación para la investigación y suincidencia en la cualificación académica de los estudiantes de Contaduría en Colombia. Teuken Bidikay(, 99- 122.

Souza . (2005). La universidad en el siglo XXI. Para una reforma democrática y emancipadora de la universidad. México: Centro de Investigaciones Interdisciplinarias en Ciencias Humanas. Mexico: Universidad Nacional Autónoma de México. 